C. Panteva

B. Ross ${ }^{\mathrm{a}}$

P. Bergb

T. Elbert ${ }^{\mathrm{b}}$

B. Rockstroh ${ }^{\mathrm{b}}$

a Center of Biomagnetism, Institute of Experimental Audiology,

University of Münster, and

${ }^{b}$ University of Konstanz, Germany

\section{Study of the Human Auditory} Cortices Using a Whole-Head Magnetometer: Left vs. Right Hemisphere and Ipsilateral vs. Contralateral Stimulation

\section{Key Words}

Hearing

Magnetoencephalogram

Auditory evoked magnetic

field

Human auditory cortex

\begin{abstract}
Structural and functional asymmetries of the temporal lobe affect language development and may also play a role in a variety of disorders, ranging from specific language impairment to schizophrenia. Whole-head neuromagnetometers allow the noninvasive measurement of functional asymmetries since activity from both hemispheres is recorded simultaneously. In the present study, the location of the auditory cortices and their responsiveness to pure tones was compared between hemispheres in healthy human subjects. Data suggest a greater contralateral than ipsilateral activation. In line with previous findings, sources of responses for the right hemisphere seem to be more anterior than for the left one.
\end{abstract}

\section{Introduction}

The region of the human auditory cortex has been defined by means of electrophysiological [Celesia, 1976] and cytoarchitectonic [Galaburda and Sanides, 1980] studies of the human brain. Viewing the lateral surface of the cortex, the auditory areas surround the more posterior part of the Sylvian fissure.
Heschl's gyrus is considered the primary auditory area. It extends in medial-posterior direction on the surface of the supratemporal plane. Based on the study of a large number of human brains, Campain and Minckler [1976] reported that the configuration of Heschl's gyrus differed between hemispheres. Some cerebral cortices contained double gyri on each side, some two on the left and one on the right 
side, and some showed a reversed asymmetry. The planum temporale is the area of the cortical surface extending from the most posterior aspect of Heschl's gyrus along the lateral fissure to the end point of the Sylvian fissure. Geschwind and Levitsky [1968] demonstrated that in humans the planum temporale was significantly larger in the left hemisphere than in the right. Furthermore, in righthanded subjects the right temporal plane extends significantly more anterior than the left temporal plane [Geschwind and Levitsky, 1968, Galaburda and LeMay, 1978; Hori, 1980].

Knowledge of the neuroanatomical asymmetries is important whenever lateral asymmetries in the functional cortical organization of the human auditory cortex are investigated. One of the key principles in the assessment of central nervous auditory function using behavioral tests is related to the lateralization of the deficit, i.e. that damage to the auditory cortex yields a deficit in the contralateral ear. This view is supported by electrophysiological evidence obtained from animal experiments [Rosenzweig, 1951], by intracortical recordings from the auditory cortex in humans during neurosurgical operations [Celesia, 1976], by EEG [Butler et al., 1969] and by magnetoencephalographic (MEG) measurements [Pantev et al., 1986; Hoke, 1988; Mäkelä, 1988; Mäkelä and Hari, 1990]. In the noninvasive EEG and MEG studies, determination of the lateralization of auditory responses was based upon the most prominent deflection of the slow auditory evoked potential or field, the N1m (M100), that evolves with a peak latency of about $100 \mathrm{~ms}$ after stimulus onset [for review see Hari, 1990]. The hemispheric dominance in healthy normal human subjects has not yet been studied in great detail, although this information would be relevant for an assessment of possible changes in this measure under pathological conditions. There are, however, a few EEG and MEG studies of auditory hemispheric dominance in normal controls [Kaukoranta et al., 1987; Berg et al., 1992, Levanen et al., 1996] and in patients with temporal lobe lesions [Woods et al., 1987, Scherg and von Cramon, 1990] and stroke [Mäkelä and Hari, 1992]. The electric dipole modelling [Berg et al., 1992] confirmed the lateral asymmetry in 23 out of 28 healthy controls and in 17 out of 24 schizophrenic patients such that $\mathrm{N} 1 \mathrm{~m}$ is centered more anteriorly on the right than on the left side. It is currently a matter of controversy whether or not schizophrenic patients exhibit deviances in this laterality measure [Rockstroh et al., 1997]. Reite et al. [1989] reported that schizophrenic patients are less likely than normal controls to show such hemispheric asymmetries.

The MEG studies cited above [Kaukoranta et al., 1987; Levanen et al., 1996; Mäkelä and Hari, 1992; Reite et al., 1989] were performed with one- or multichannel neuromagnetometers that could measure responses from only one hemisphere at a time. This is a serious drawback, as the evoked brain activity then depends on the repetition of measurements and on variables such as vigilance and arousal, while whole-head MEG systems allow the comparison of simultaneously measured responses from both hemispheres and the evaluation of laterality as a consequence of side of stimulation. To our knowledge only two studies of functional differences between the two auditory cortices in normal human subjects are based on whole-head neuromagnetic recordings: the first [Mäkelä et al., 1993] was carried out with 122 planar gradiometer channels, the second [Nakasato et al., 1995] with 66 axial gradiometer channels. The goal of the present study, performed with 148 magnetometer channels covering the whole head, was to obtain data from healthy human subjects about interhemispheric differences and lateralization with respect to the side of stimulation. 


\section{Methods}

Auditory evoked magnetic responses to pure tones were measured using a whole head neuromagnetometer (Magnes 2500, BTi). Two female and 4 male subjects aged between 30 and 50 years (mean age 37 years) with no history of otological or neurological disorders participated in the study. A normal audiological status was assured in that air conduction and bone conduction thresholds of no more than $10 \mathrm{~dB}$ hearing level in the range from 250 to $8,000 \mathrm{~Hz}$ were allowed. All subjects were right-handed according to the Edinburgh handedness questionnaire [Oldfield, 1971]. Informed consent was obtained from each subject after explaining to her/him the nature of the study. Subjects were paid for their participation. The data of 2 additional subjects served as pilot investigations to test the new measurement system and were not included in the subsequent analyses.

Auditory stimuli comprised five different tone bursts (duration $500 \mathrm{~ms}, 10-\mathrm{ms}$ rise and fall time; cosine slope) with frequencies of $250,500,1,000,2,000$ and $4,000 \mathrm{~Hz}$. The slope was sufficiently steep to elicit a prominent $\mathrm{N} 1 \mathrm{~m}$ component in the auditory evoked field (AEF), but gradual enough to retain a narrow frequency spectrum. Stimulus intensity was set to $60 \mathrm{~dB}$ relative to the individual hearing threshold. The interstimulus interval varied randomly between 2.7 and $3.3 \mathrm{~s}$. For each of the five frequencies, 128 stimuli constituted one train of stimuli. The series of five stimulus blocks was delivered to the left and to the right ear, the sequence of ears and frequencies varying pseudorandomly across subjects. The magnetically silent delivery of the stimuli was realized by means of a special delivery system with speakers (compressor driver type) outside the magnetically shielded room and an echo-free tone transmission through a plastic tube $6.3 \mathrm{~m}$ in length and $16 \mathrm{~mm}$ in inner diameter into a silicon ear piece. This system provided almost linear frequency characteristics in the range between 200 and $4,000 \mathrm{~Hz}$ (deviations less than $\pm 4 \mathrm{~dB}$ ) [Pantev et al., 1991].

Auditory magnetic fields evoked by the different stimuli were recorded simultaneously from the left and the right hemispheres. The measuring surface of the whole head sensor is helmet-shaped and covers the entire cranium. An indent at the ears ensures patient comfort, closer coil-to-head spacing and room for auditory stimulus delivery. Within the whole head sensor 148 signal detectors (magnetometer-type) are arranged in a uniformly distributed array spaced out $28 \mathrm{~mm}$ apart. A supine measurement position was chosen as being more comfortable and ensuring that the subject did not move during the measurement. Special care was taken to ensure that the subject's head was positioned in the middle of the helmet. The stability of the head-sensor position was controlled by repeated measurement of the known positions of five indicator coils fixed on the scalp. The subjects were instructed not to move and to stay in a relaxed waking state during the measurement. Compliance was verified by video-monitoring throughout the measurement.

Stimulus-related epochs of $1,000 \mathrm{~ms}$ (including prestimulus time of $200 \mathrm{~ms}$ ) were recorded with a bandwidth of $1-100 \mathrm{~Hz}$ and a sampling frequency of 387.5 $\mathrm{Hz}$. The auditory event-related field (AEF) that was submitted to source analysis resulted from an average of approximately 128 stimulus epochs. Epochs contaminated by muscle or eye blink artifacts with amplitude variations of more than $3 \mathrm{pT}$ in any channel were automatically rejected from the averaging procedure.

In order to compare these first measurements with the BTi Magnes whole-head system with results obtained from recordings with a limited number of channels over one hemisphere, a single equivalent current dipole (ECD) in a best fitting local sphere was estimated separately for the left and the right hemisphere for each stimulus condition, although there is some experimental evidence for two or more $\mathrm{N} 1 \mathrm{~m}$ equivalent sources with different locations [Williamson et al., 1991; Moran et al., 1993; Cansino et al., 1994]. Since the AEF generated in the left and the right hemisphere showed little overlap (fig. 1), it was feasible to select subsets of about 40 channels that include the signal from either the left or the right auditory areas for source analysis. An ECD defined by dipole moment, orientation and space coordinates was calculated for each sample point. The location of the ECD was estimated in a head-based coordinate system. The origin of this coordinate system was set at the midpoint of the mediallateral axis (y axis) which joined the center points of the entrance to the acoustic meatus of the left and the right ears (positive towards the left ear). The posterior-anterior axis ( $\mathrm{x}$ axis) was oriented from the origin to the nasion (positive towards the nasion) and the inferiorsuperior axis ( $\mathrm{z}$ axis) was perpendicular to the $\mathrm{x}-\mathrm{y}$ plane (positive towards the vertex). Further analyses of the experimental data were concentrated on the major component of the AEF, the N1m. Each N1m dipole parameter was represented by the average of twelve data points (30-ms interval) around the maximum of the root mean square of the magnetic field calculated across the respective subsets of channels. The calculated values were accepted for further analysis when they satisfied the following source analysis and anatomical requirements: (1) goodness of fit of the ECD model to the measured field greater than $90 \%$; (2) confidence 


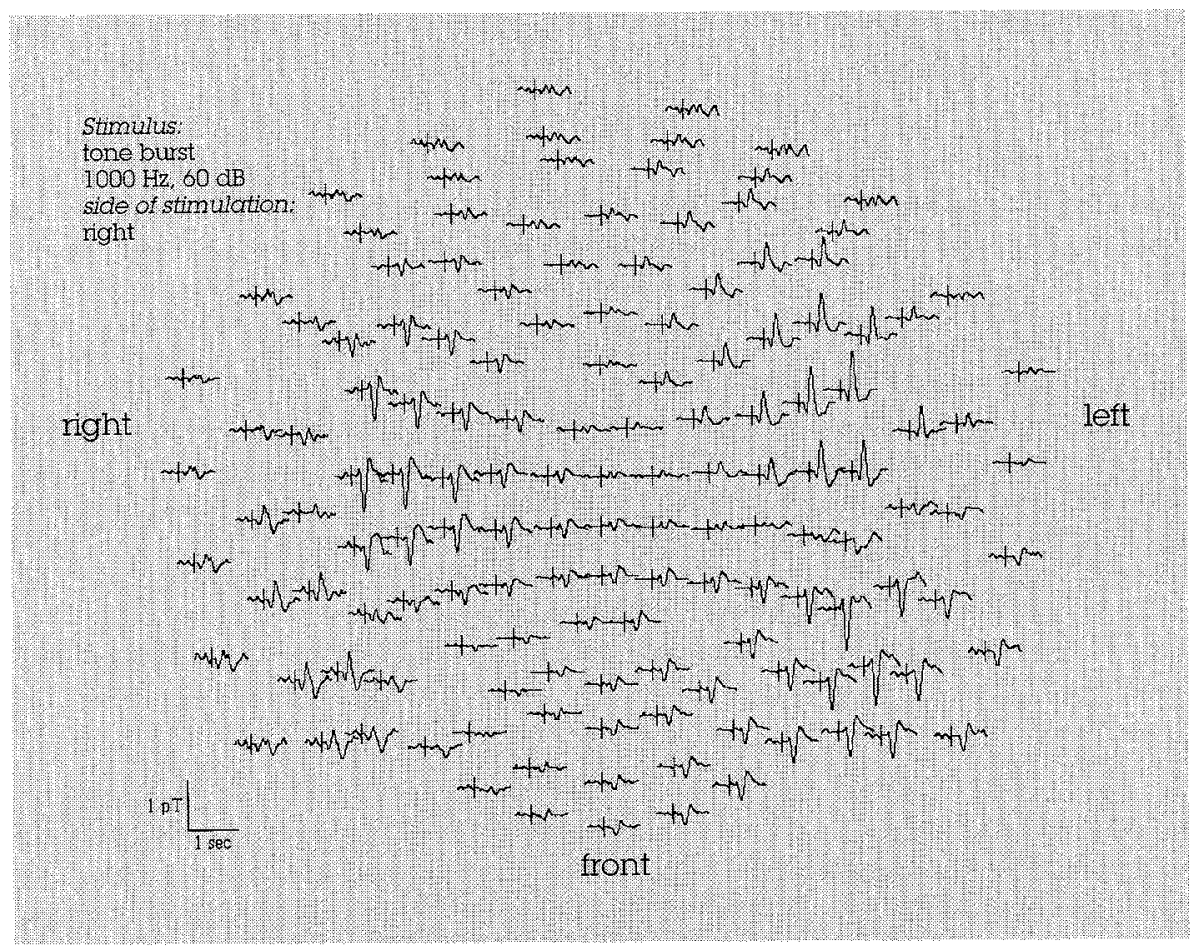

Fig. 1. AEF of 1 subject across the sensor array elicited by right-ear tone burst stimulation with a carrier frequency of $1,000 \mathrm{~Hz}$ and intensity of $60 \mathrm{~dB} \mathrm{SL}$.

volume less than $300 \mathrm{~mm}^{2}$; (3) range of the source coordinates within the $300-\mathrm{ms}$ interval of less than $2 \mathrm{~cm}$; (4) anterior-posterior value within $\pm 3 \mathrm{~cm}$; medial-lateral value (distance to the midsagittal plane) greater than $2.5 \mathrm{~cm}$, and inferior-superior value greater than $3 \mathrm{~cm}$ and less than $8 \mathrm{~cm}$. The individual median was calculated across those stimulation frequencies that met the above-listed requirements. Thus, for each measure and subject a score was obtained for the side of stimulation (ipsi- and contralateral) and the two hemispheres (right and left). The statistical analysis compared the effects of side of stimulation and of hemisphere for the median across frequencies. For this purpose, the calculated three-dimensional dipole source locations, the dipole moment $\mathrm{q}$ and the root mean square across channels (rms) were submitted to analyses of variance (repeated measurement) with the factors Side of Stimulation (ipsi- vs. contralateral) and Hemisphere (left vs. right). In addition, nonparametric one-sample sign tests were performed comparing hemispheres (LH-RH) and ipsiand contralateral side of stimulation (I-C).

\section{Results}

Figure 1 illustrates the AEF for 1 subject across the sensor array elicited by right-ear stimulation with the $1,000-\mathrm{Hz}$ tone burst. Over each of the hemispheres, a dipolar structure can be observed for the major AEF component $\mathrm{N} 1 \mathrm{~m}$. Closer inspection of the figure reveals larger amplitudes over the left hemisphere: The N1m amplitude (referred to prestimulus baseline) is about $10 \%$ larger and the N1m latency is about $6 \%$ shorter in the contralateral left hemisphere than in the ipsilateral right hemisphere. The reversed asymmetry was observed for left-ear stimulation.

An example of the source analysis based on the data of the subject presented in figure 1 is displayed in figure 2 . In this case, the good- 

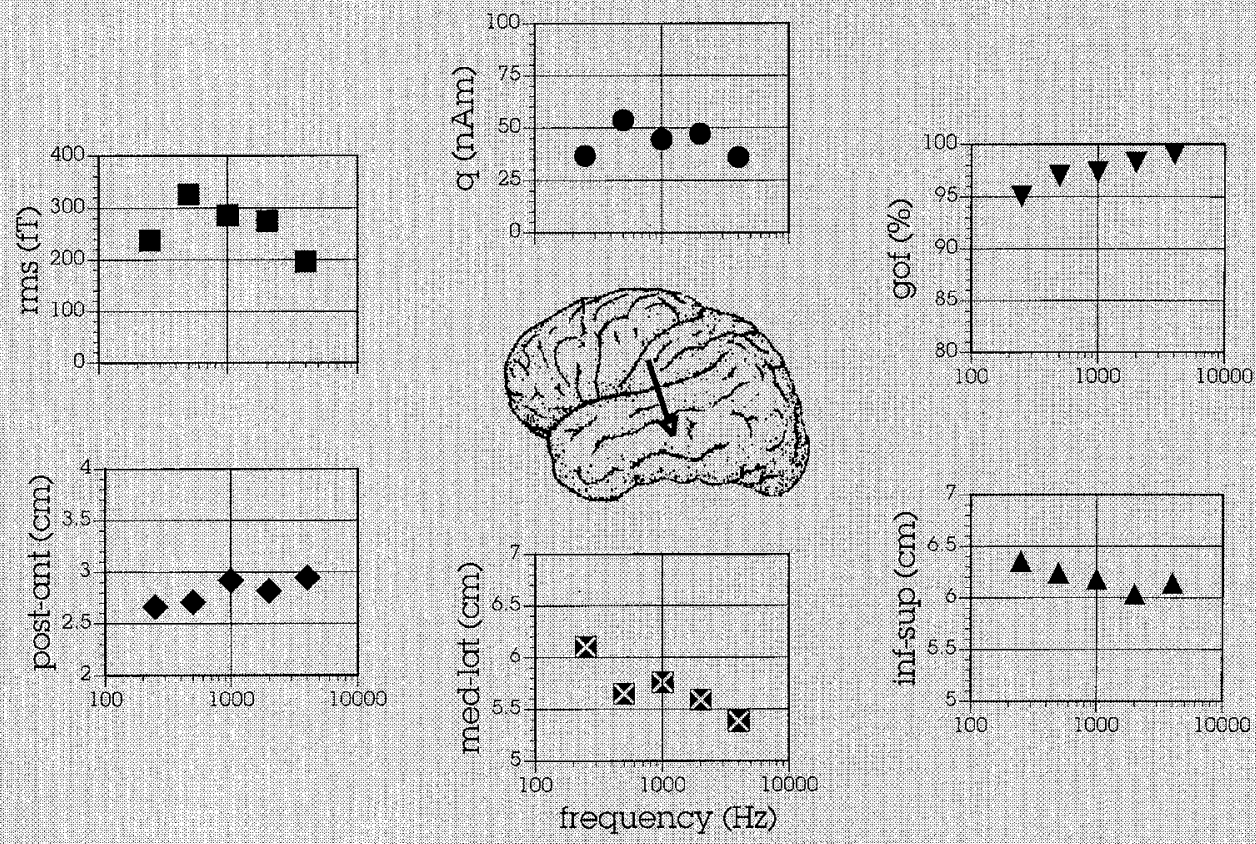

Fig. 2. Example of the source analysis based on the data presented in figure 1 . The schematic representation in the center of the figure suggests the location of the equivalent source as projected onto the lateral surface of the brain.

ness of fit for the analysis of the data from the left hemisphere (right-ear stimulation) was better than $95 \%$ for each of the five stimulation frequencies. With increasing stimulus frequency the determined source locations shift primarily in medial but also somewhat in anterior and inferior direction.

Statistical results of this study are summarized in figure 3 . Figure $3 \mathrm{c}$ is helpful to understand the comparisons made with respect to the side of stimulation and hemisphere. Both the root mean square value of the measured field strength (rms) and the absolute value of the estimated dipole moment (q) are larger for the contralateral hemisphere (main effect of Side of Stimulation for rms: $F(1,5)=16.8, p<$
0.01 ; for $\mathrm{q}: \mathrm{F}(1,5)=7.4, \mathrm{p}<0.05)$. The rms is $80 \mathrm{fT}$ and $\mathrm{q}$ is $13 \mathrm{nA}$ larger for contralateral as compared to ipsilateral stimulation (fig. 3a, b). There is virtually no rms difference between the left and the right hemispheres (fig. 3a). In contrast, the dipole moment is $7 \mathrm{nA}$ larger in the right hemisphere as compared to the left hemisphere (fig. $3 \mathrm{~b}$; main effect of Hemisphere, $F(1,5)=3.2$, n.s.).

No significant differences were observed for the spatial coordinates of the equivalent source in the medial-lateral or in the inferiorsuperior direction (fig. $3 \mathrm{e}, \mathrm{f}$ ), except the pronounced tendency of the N1m sources to be more medial in the left as compared to the right hemisphere. In 5 of the 6 subjects, the 


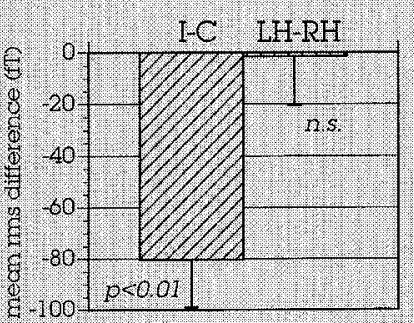

a.

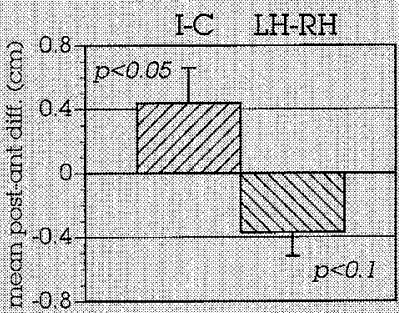

a

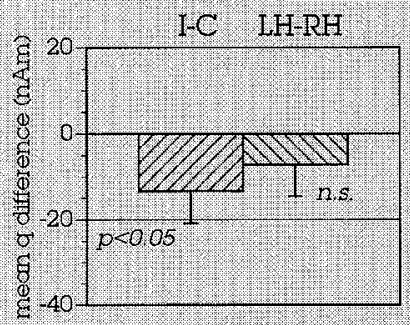

16.

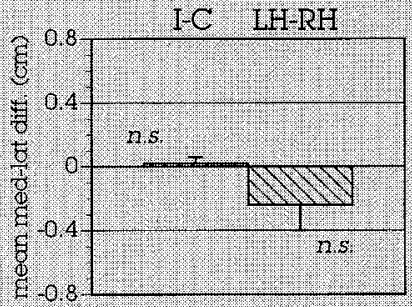

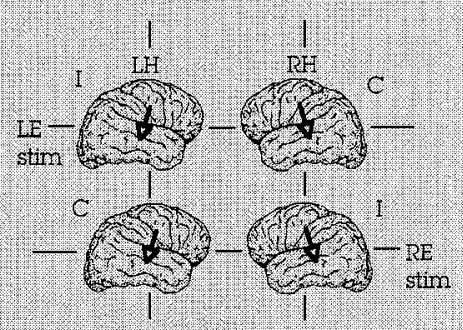

(9.

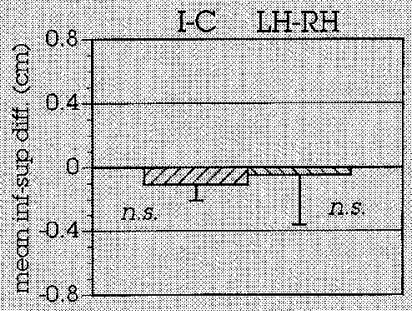

Fig. 3. a, b Ipsilateral-contralateral (I-C) and left-right hemisphere (LH-RH) comparisons for the field strength and dipole moment. c Schematic illustration of these comparisons. d, e, f I-C and LH-RH differences for the spatial source coordinates.

$\mathrm{N} 1 \mathrm{~m}$ sources were $2-8 \mathrm{~mm}$ (median $4 \mathrm{~mm}$ ) more anterior on the right than on the left side. One subject, however, showed a reversed asymmetry of $2 \mathrm{~mm}$ (the main effect of Hemisphere, with $F(1,5)=5.8, p=0.06$ is significant only when a one-tailed test is assumed; such an assumption is justified, as it was predicted on the basis of previous anatomical and functional asymmetries). In all 6 subjects, sources were somewhat more anterior for the ipsi- than for the contralateral stimulation ( $p<0.05$ for the sign test; fig. 3d). For the cases where a complete data set was available, this difference in right/left ear representation could be observed for each of the single frequencies.

\section{Discussion}

The shorter latency (mean difference of $7 \mathrm{~ms}$ ) of the contralateral N1m peak that we have obsèrved in this study is in line with previous results in single-hemisphere MEG studies [Elberling et al., 1980; Pantev et al., 1986; Mäkelä, 1988; Rogers et al., 1990] as well as whole-head MEG [Mäkelä et al., 1993; Nakasato et al., 1995]. This result corresponds to the anatomical differences of the auditory ascending pathways, being shorter after the colliculi inferior on the contralateral than on the ipsilateral side [Evans, 1982]. Also the significantly larger rms field value to contralateral stimulation confirms the findings of the studies cited above. A reasonable explanation of this effect is that most of the fibers of the audi- 
tory pathway are crossing to the contralateral side where a larger cortical response is evoked. These findings are also consistent with previous investigations based on source modelling of EEG data: Scherg and von Cramon [1986] report that the electrical dipole source in the contralateral temporal lobe is about $10 \%$ larger and develops earlier as compared to the ipsilateral side.

The observed hemispheric asymmetry of the N1m source location has also been reported in EEG [Berg et al., 1992] and in MEG studies [Hoke, 1988; Mäkelä et al., 1993; Nakasato et al., 1995]. In the majority of our subjects, with one exception, the sources were more anterior in the right hemisphere than in the left hemisphere. The functional asymmetry estimated in the MEG-source analysis correlates with the anatomical asymmetry of the human temporal lobe. The lateral part of Heschl's gyrus and the temporal plane, i.e. the probable generator sites of the N1 m [LiégeoisChauvel et al., 1994; Pantev et al., 1995], are located more anteriorly on the right-hemisphere for right-handed subjects [Geschwind and Levitsky, 1968; Galaburda and LeMay, 1978].

The finding that the $\mathrm{N} 1 \mathrm{~m}$ sources were located more anterior for the ipsi- than for contralateral stimulation was unexpected. The effect, although quite small, was observed consistently in all of the subjects. It suggests that the representations of the two different basilar membranes occupy different portions of auditory cortex, and may point towards an alternating dominance of right and left ear representation in the auditory cortical tonotopic map, possibly comparable to the right and left dominance columns in the visual system. Although such a pattern was also found for the different stimulation frequencies within most of the subjects, the present data base is too small to provide strong evidence for such a speculation.

\section{Acknowledgments}

This work was supported by grants from the Deutsche Forschungsgemeinschaft Pa 392/6-1, 6-2 and El 101/13.

\section{References}

Berg P, Schrodt A, Cohen R, Scherg M: Multiple source analysis of the auditory N1M00 in schizophrenics and healthy controls. Proc 10th Int Conf on Event-Related Potential Research, Eger, 1992, p 14.

Butler R, Keidel W, Spreng M: An investigation of the human cortical evoked potential under conditions of monaural and binaural stimulation. Acta Otolaryngol 1969;68: 317-326.

Campain R, Minckler J: A note on gross configurations of the human auditory cortex. Brain Lang 1976;3:318323.
Cansino S, Williamson S, Karron D: Tonotopic organization of the human auditory association cortex. Brain Res 1994;663:38-50.

Celesia GG: Organization of auditory cortical areas in man. Brain 1976; 99:403-414.

Elberling C, Bak C, Kofoed B, Lebech C, Saermark K: Magnetic auditory responses from the human brain. A preliminary report. Scand Audiol 1980;9:185-190.

Evans EF: Functional anatomy of the auditory system; in Barlow $\mathrm{HB}$, Mollon JD (eds): The Senses. Cambridge, Cambridge University Press, 1982, pp 255-306.
Galaburda A, Sanides F: Cytoarchitectonic organization of the human auditory cortex. J Comp Neurol 1980; 190:597-610.

Galaburda AM, LeMay M: Right-left asymmetries in the brain. Science 1978; 199:852-856.

Geschwind N, Levitsky W: Human brain: Left right asymmetries in temporal speech region. Science 1968;161:186-187.

Hari R: The neuromagnetic method in the study of the human auditory cortex; in Grandori F, Hoke M, Romani GL (eds): Auditory Evoked Magnetic Fields and Electric Potentials. Adv Audiol. Basel, Karger, 1990, vol 6, pp 222-282. 
Hoke M: Auditory evoked magnetic fields; in Basar E (eds): Dynamics of Cognitive and Sensory Processing by the Brain. Berlin, Springer, 1988, pp 311-318.

Hori $\mathrm{T}$ : Hemispheric asymmetry in man. Shinkei Shimpo 1980;24:479496.

Kaukoranta E, Hari R, Lounasmaa OV: Responses of the human auditory cortex to vowel onset after fricative consonants. Exp Brain Res 1987;69: 19-23.

Levanen S, Ahonen A, Hari R, McEvoy L, Sams M: Deviant auditory stimuli activate human left and right auditory cortex differently. Cereb Cortex 1996;6:288-296.

Liégeois-Chauvel C, Musolino A, Badier JM, Marquis P, Chauvel P: Evoked potentials recorded from the auditory cortex in man: Evaluation and topography of the middle latency components. Electroencephalogr Clin Neurophysiol 1994;92:204214.

Mäkelä JP: Contra- and ipsilateral auditory stimuli produce different activation patterns at the human auditory cortex: A neuromagnetic study. Pflügers Arch 1988;412:12-16.

Mäkelä JP, Ahonen A, Hämäläinen M, Hari R, Ilmoniemi R, Kajola $M$, Knuutila J, Lounasmaa OV, McEvoy $L$, Salmelin $R$, et al: Functional differences between auditory cortices of the two hemispheres revealed by whole-head neuromagnetic recordings. Hum Brain Mapping 1993;1:48-56.

Mäkelä JP, Hari R: Long-latency auditory evoked magnetic fields. Adv Neurol 1990;54:177-191.
Mäkelä JP, Hari R: Neuromagnetic auditory evoked responses after a stroke in the right temporal lobe. Neuroreport 1992;3:94-96.

Moran JE, Tepley N, Jacobson GP, Barkley GL: Evidence for multiple generators in evoked responses using finite difference field mapping: Auditory evoked fields. Brain Topogr 1993;5:229-240.

Nakasato N, Fujita S, Seki K, Kawamura T, Matani A, Tamura I, Fujiwara S, Yoshimoto T: Functional localization of bilateral auditory cortices using an MRI-linked whole head magnetencephalography (MEG) system. Electroencephalogr Clin Neurophysiol 1995;94:183-190.

Pantev C, Bertrand O, Eulitz C, Verkindt C, Hampson S, Schuirer G, Elbert T: Specific tonotopic organizations of different areas of the human auditory cortex revealed by simultaneous magnetic and electric recordings. Elecroencephalogr Clin Neurophysiol 1995;94:26-40.

Pantev C, Gallen C, Hampson S, Buchanan S, Sobel D: Reproducibility and validity of neuromagnetic source localization using a large array biomágnetometer. Am J EEG Technol 1991;31:83-101.

Pantev C, Lütkenhöner B, Hoke M, Lehnertz $\mathrm{K}$ : Comparison between simultaneously recorded auditoryevoked magnetic fields and potentials elicited by ipsilateral, contralateral and binaural tone burst stimulation. Audiology 1986;25:54-61.

Reite M, Teale P, Goldstein L, Whalen J, Linnville S: Late auditory magnetic sources may differ in the left hemisphere of schizophrenic patients: A preliminary report. Arch Gen Psychiatry 1989;46:565-572.
Rockstroh B, Elbert T, Berg P: Die Untersuchung von Gehirnfunktionen in der experimentellen Psychopathologie am Beispiel der Schizophrenie; in Rockstroh B, Elbert T, Watzl $H$ (eds): Impulse für die Klinische Psychologie. Göttingen, Hogrefe, 1997, pp 1-27.

Rogers RL, Papanicolaou AC, Baumann SB, Eisenberg HM, Saydjari C: Spatially distributed cortical excitation patterns of auditory processing during contralateral and ipsilateral stimulation. J Cogn Neurosci 1990; 2:44-50.

Rosenzweig N: Representation of the two ears at the auditory cortex. Am J Physiol 1951;167:147-158.

Scherg M, von Cramon D: Evoked dipole source potentials of the human auditory cortex. Electroencephalogr Clin Neurophysiol 1986;65:344360.

Scherg M, von Cramon D: Dipole source potentials of the auditory cortex in normal subjects and in patients with temporal lobe lesions; in Grandori F, Hoke M, Romani GL (eds): Auditory Evoked Magnetic Fields and Electric Potentials. Adv Audiol. Basel, Karger, 1990, vol 6, pp 165193.

Williamson SJ, Lu ZL, Karron D, Kaufman L: Advantages and limitations of magnetic source imaging. Brain Topogr 1991;4:169-180.

Woods DL, Clayworth CC, Knight RT, Simpson GV, Naeser MA: Generators of middle and long-latency auditory evoked potentials: Implications from studies of patients with bitemporal lesions. Electroencephalogr Clin Neurophysiol 1987;68: 132-148. 